\title{
Pulmonary nodule radiological diagnostic algorithm in lung cancer screening
}

\author{
Katarzyna Dziadziuszko, Edyta Szurowska \\ II Department of Radiology, Medical University of Gdańsk, Gdańsk, Poland \\ Contributions: (I) Conception and design: All authors; (II) Administrative support: All authors; (III) Provision of study materials or patients: All \\ authors; (IV) Collection and assembly of data: All authors; (V) Data analysis and interpretation: All authors; (VI) Manuscript writing: All authors; (VII) \\ Final approval of manuscript: All authors. \\ Correspondence to: Edyta Szurowska. II Department of Radiology, Medical University of Gdańsk, 17 Smoluchowskiego St., 80-214 Gdańsk, Poland. \\ Email: eszurowska@gumed.edu.pl.
}

\begin{abstract}
Publications of the final results of the two largest randomized lung cancer screening (LCS) trials in the United States and Europe confirmed the reduction in the mortality rate associated with the use of screening with low-dose computed tomography (LDCT). Results of these trials led to widespread acceptance of LCS in properly defined high-risk populations, and its implementation in the clinical practice. Many countries started preparation for national LCS and refreshed still open debate about lung nodule management. Detection of lung cancer in the early stage with a reduction of lung cancer mortality requires dedicated programs with optimized protocols, including a specified pulmonary nodule diagnostic algorithm. The screening protocol should be clear with a precise nodule diameter or volume threshold, based on which a positive screen result is defined. The application of risk-prediction models and the introduction of the semiautomated assessment of detected nodules improves screening accuracy and should be applied in LCS protocols as verified tools to aid radiological diagnosis. In this review, we have summarized recent data about the radiological protocols from the most important LCS programs and pulmonary diagnostic algorithms. These protocols should be taken into consideration in the ongoing and planned LCS programs.
\end{abstract}

Keywords: Lung cancer (LC); screening; pulmonary nodule

Submitted Jun 17, 2020. Accepted for publication Nov 23, 2020.

doi: $10.21037 /$ tlcr-20-755

View this article at: http://dx.doi.org/10.21037/tlcr-20-755

\section{Introduction}

Five-year survival of patients with diagnosed lung cancer (LC) does not exceed $18 \%$ and has not changed significantly over the past few decades (1). Improvements in these statistics are expected to result from large-scale screening programs using low-dose computed tomography (LDCT), as demonstrated in several milestone publications, including two large, randomized LC screening (LCS) trials that documented a reduction of LC mortality $(2,3)$. Based on experience from LCS trials, societies and research teams proposed different algorithms for assessment of pulmonary nodules detected in chest CTs which evolved through the years. A lung nodule, as defined by the Fleischner Society, is a focus rounded or irregular opacity, which may be well or poorly defined with a diameter $\leq 3 \mathrm{~cm}$, surrounded by lung parenchyma on radiological imaging (4). Radiological assessment of lung nodules on LDCT performed according to protocols of LCS should begin with the qualification of the nodule to the appropriate group, and then, depending on its type and size, further diagnostic procedures or the next round of LDCT assessment (5). The nodules should be categorized into four different groups according to their density: solid, non-solid, partly solid, or calcified. A solid nodule is homogeneous and presents soft-tissue attenuation. A non-solid nodule is like a pure ground-glass nodule (GGN) and does not obstruct the structure of the lung parenchyma. A partly solid nodule has both solid and ground-glass 
components. A calcified nodule contains calcifications, which are easily identified on CT. Size, volume, and growth rate are the most important discriminators for the probability of nodule malignancy (6). Different approaches were practiced according to the evaluation of these features. In this review, we summarized main LCS protocols focusing on proposed cut-offs and further management of the detected nodules.

\section{Early Lung Cancer Action Project (ELCAP)}

The ELCAP was designed in New York in 1992 to evaluate the benefits of annual CT LCS. ELCAP was the first singlearm prospective study involving 1000 volunteers, aged 60 years and older with a history of smoking of at least 10 pack-years. Each patient received baseline chest radiographs (CXR) in two projections and LDCT (7). Positive scan results were defined by the presence of one to six noncalcified pulmonary nodules (NCNs) on LDCT images, regardless of their size $(8,9)$. When no NCN was found, the result was negative. Detection of more than $6 \mathrm{NCNs}$ or diffuse bronchiectasis, ground-glass opacities (GGOs), or a combination of them was considered to be a diffuse disease. Further recommendations included a high-resolution CT (HRCT) follow-up for nodules $<10 \mathrm{~mm}$ three months later, to assess the nodule's growth rate. When no growth was observed, a subsequent CT was performed after 6 , 12 , and 24 months. The stable size of the nodule within 2 years was considered to confirm its benign nature, and the patient returned to annual screening. If the NCN growth rate indicated a potential malignant change, a biopsy was performed immediately. A biopsy and/or bronchoscopy was also the test of choice in nodules of $10 \mathrm{~mm}$ and more.

If HRCT showed benign calcifications in a nodule $<20 \mathrm{~mm}$ with smooth edges, the nodule was considered benign. Data from the baseline ELCAP study indicate that positive CT results were obtained 3 times more frequently than those based on CXR (23\% vs. 7\%). When using CT vs. CXR, lung cancer (LC) was detected 4 times more often $(2.7 \%$ vs. $0.7 \%)$ and 6 times more often in stage I $(2.3 \%$ vs. $0.4 \%) .233$ out of 1,000 participants had a positive CT scan, of which 27 patients (12\%) had confirmed LC, which was detectable on CXR in only 7 cases (10). This study showed that LDCT screening enables the detection of LC at an early stage with a higher cure rate than that of symptomatic LC.

\section{NY-ELCAP}

ELCAP was further expanded to New York State (NY-
ELCAP), and between 2000-2003, 6,295 participants with a smoking history of 40 pack-years were recruited; 5,134 patients had one annual round of screening after a year, and 880 participants two annual rounds $(11,12)$. Compared to ELCAP, modern CT scanners with a thinner 1.25 vs. $10.0 \mathrm{~mm}$ acquisition layer and CT workstations instead of film assessment have been used $(11,13)$. Screening examinations were considered positive when solid and/or part-solid nodules with a diameter $\geq 5 \mathrm{~mm}$ and/or non-solid nodules with a diameter $\geq 8 \mathrm{~mm}$ were detected; semi-positive when solid and/or part-solid nodules of a size $<5 \mathrm{~mm}$ and/or nonsolid nodules $<8 \mathrm{~mm}$ were found (12). Negative results meant no nodules. Introduction of a new screening protocol with the fast development of CT technology allowed for the detection of very small nodules of $2 \mathrm{~mm}$, leading to an increased percentage of false-positive results with thresholds set too low (Table 1).

\section{International Early Lung Cancer Action Project (I-ELCAP)}

Further expansion of the ELCAP outside the US, among others, to Canada, Israel, Italy, Spain, and Switzerland, transformed it into the I-ELCAP and provided sufficient data to reliably estimate the probability of survival in LC patients as part of their screening (initial round, annual rounds, as well as diagnostic rounds, due to symptoms).

The first results of I-ELCAP, published in 2006, showed 10 -year survival probability of $88 \%$ (95\% CI, 84-91). Patients with stage I LC and with LC resected within 1 month from diagnosis had a higher survival of $92 \%$.

These results were published from data collected from 31,567 participants, among whom 484 were diagnosed with LC $(1.5 \%)$. The majority of cancers (85\%) were in Stage I (14). The screening protocol used to obtain these results was similar to the NY-ELCAP study. Positive baseline screening results with thresholds of $5 \mathrm{~mm}$ for solid and part-solid nodules and $8 \mathrm{~mm}$ for non-solid nodules were established (12) (Table 1).

In the annual repeat screening, positive results were defined as new NCNs regardless of their size, or nodules which show the features of growth in relation to the baseline scans. The follow-up algorithm was: solid or part-solid nodules of size 1-4 mm-follow-up LDCT after 6 months; solid or part-solid nodules of size $\geq 5 \mathrm{~mm}$, or non-solid nodule $\geq 8 \mathrm{~mm}$-antibiotic therapy and LDCT after one month; for all other cases, positron emission tomography (PET) or nodule biopsy was recommended $(15,16)$. 
Table 1 False positive rate (FPR) and different thresholds for negative, intermediate and positive screen at baseline in lung cancer screening programs

\begin{tabular}{|c|c|c|c|c|c|c|c|c|c|c|c|}
\hline Program & $\begin{array}{c}\text { Recruitment } \\
\text { period }\end{array}$ & FPR & \multicolumn{3}{|c|}{ Negative } & \multicolumn{3}{|c|}{ Intermediate } & \multicolumn{3}{|c|}{ Positive } \\
\hline NY-ELCAP & 2002-2003 & $70 \%$ & $\begin{array}{c}\text { No } \\
\text { nodules }\end{array}$ & & & $<5 \mathrm{~mm}^{(\mathrm{II})}$ & $<5 \mathrm{~mm}^{(\mathrm{II})}$ & $<8 \mathrm{~mm}^{(\mathrm{II})}$ & $\geq 5 \mathrm{~mm}^{(\mathrm{II})}$ & $\geq 5 \mathrm{~mm}^{(\mathrm{II})}$ & $\geq 8 \mathrm{~mm}^{(\text {II) }}$ \\
\hline \multirow[t]{2}{*}{ I-ELCAP } & 2000-2011 & $\mathrm{n} / \mathrm{a}$ & $\begin{array}{c}\text { No } \\
\text { nodules }\end{array}$ & & & $<5 \mathrm{~mm}^{(\mathrm{II})}$ & $<5 \mathrm{~mm}^{(I I)}$ & $<8 \mathrm{~mm}^{(\mathrm{II})}$ & $\begin{array}{c}\geq 5 \mathrm{~mm}^{(\mathrm{II})} \text { or } \\
\text { endobronchial } \\
\text { nodule (any } \\
\text { size) }\end{array}$ & $\geq 5 \mathrm{~mm}^{(\text {III) }}$ & $\geq 8 \mathrm{~mm}^{(\mathrm{II})}$ \\
\hline & 2012- & $\mathrm{n} / \mathrm{a}$ & $\begin{array}{c}\text { No } \\
\text { nodules }\end{array}$ & & & $\begin{array}{c}<6 \mathrm{~mm}^{(I I)} ; \\
6-14.9 \mathrm{~mm}^{(I I)}\end{array}$ & $\begin{array}{c}\text { Solid } \\
\text { component } \\
<6 \mathrm{~mm}^{(\mathrm{II})} ; \\
\text { solid component } \\
6-14.9 \mathrm{~mm}^{(\mathrm{II})}\end{array}$ & Any size & $\begin{array}{c}\geq 15 \mathrm{~mm}^{(\mathrm{II})} \text { or } \\
\text { endobronchial } \\
\text { nodule (any } \\
\text { size) }\end{array}$ & & \\
\hline UKLS & 2011-2014 & $3.6 \%$ & $<15 \mathrm{~mm}^{3}$ & & & $\begin{array}{r}15-49 \mathrm{~mm}^{3} \text {; } \\
50-500 \mathrm{~mm}^{3}\end{array}$ & $\begin{array}{c}<5 \mathrm{~mm}^{(I)} \text { or solid } \\
\text { component: } \\
<15 \mathrm{~mm}^{3} \\
\text { solid component: } \\
15-500 \mathrm{~mm}^{3} \text { or } \\
\text { non-solid } \\
\text { component: } \\
\geq 5 \mathrm{~mm}^{(I I)}\end{array}$ & $\geq 5 \mathrm{~mm}^{(\mathrm{II})}$ & $>500 \mathrm{~mm}^{3}$ & $\begin{array}{c}\text { Solid } \\
\text { component: } \\
>500 \mathrm{~mm}^{3}\end{array}$ & \\
\hline
\end{tabular}

(I), maximum diameter; (II), mean diameter. False positive rate (FPR) varies among different trials due to different definitions and are not directly comparable.

Nodule size in I-ELCAP was defined as the average of its length and width on a single axial image. The positive result threshold has been constantly updated since the beginning of the I-ELCAP in 2000, due to the progress of technology, acquired experience, and gathering new evidence $(9,15-18)$. Based on data received from the baseline LDCT scans of 21,136 participants of I-ELCAP, the frequency of positive results was $16 \%(3,396 / 21,136)$ for a threshold size of $5 \mathrm{~mm}$ (17). If alternative cutoffs between 6 to $9 \mathrm{~mm}$ were used, the rates of positive results decreased from $10.2 \%$ to $4.0 \%$ and cancer diagnosis would be delayed for 9 months retrospectively for $0 \%$ and $6.7 \%$ of people with LC, leading to the reduction of work-up by $36 \%$ and $75 \%$.

The I-ELCAP protocol was subsequently updated in
2012. In the baseline LDCT scans, the cut-off is $6 \mathrm{~mm}$ for solid nodules and solid fraction of part-solid nodules, and $3 \mathrm{~mm}$ for new nodules detected in the next rounds $(19,20)$. Solid and part-solid NCNs with a diameter $\geq 6 \mathrm{~mm}$ require further diagnostics-a follow-up LDCT after 3 months, biopsy or PET (for NCN $\geq 10 \mathrm{~mm}$ ). For nodules $\geq 15 \mathrm{~mm}$ with a suspicion of infection, antibiotic therapy with a followup CT in 1 month is recommended. For non-solid nodules of any size, annual LDCT screening is indicated (20). A solid endobronchial nodule needs a CT scan after 1 month.

If at least one of the new solid nodule or solid components of part-solid NCNs has a mean diameter of $3-5.9 \mathrm{~mm}$ in the annual round, a CT scan after 6 months 
should be performed, and if growth is at a malignant rate, a biopsy is needed; for solid NCN $\geq 6.0 \mathrm{~mm}$, immediate antibiotic therapy with a follow-up CT 1 month later is recommended. A lack of nodule regression after treatment is an indication for biopsy or PET (for NCN $\geq 10 \mathrm{~mm}$ ) (20).

Screening studies showed differences in the growth rate of diagnosed tumors between the initial screening test and subsequent annual rounds: the LCs detected in the baseline showed slower growth than those detected in repeated annual screening rounds and between rounds $(21,22)$. Nodules detected in annual screening rounds have a comparable growth rate to those detected outside of screening (23).

Nodule growth on CT scans can manifest as an enlargement of the entire nodule irrespective of its consistency, enlargement of the solid part in a partsolid nodule, and increase in its attenuation in nonsolid nodules (24-26). Growth can be monitored in two ways-by measuring the nodule diameter or volume with volume-doubling times (VDT), VDT measurement can be influenced by the nodule properties, the scanner type, and parameters and the model of software $(27,28)$. Despite these limitations, VDTs are a useful tool to predict a nodule malignant character.

The VDT of LC depends on its histopathological type. The recommended value of VDTs for use in screening as a predictor for LC is between 30 and 400 days. The average VDT for LC is 120-180 days with a lower VDT for small cell carcinoma. $(29,30)$ and $<30$ days for infection (31). Much higher VDTs can be observed in adenocarcinomas manifesting as non-solid and part-solid nodules $(23,32)$.

The benefit of "watchful waiting" for non-solid NCNs was gained during the I-ELCAP project and subsequently incorporated into the revised I-ELCAP protocol to avoid overtreatment (13). Due to annual LDCT followups of non-solid and part-solid NCNs, all diagnosed adenocarcinomas in I-ELCAP were $100 \%$ curable $(33,34)$. These observations led to a change in the pathological classification of adenocarcinoma and the revision of the World Health Organization classification $(35,36)$. In addition, ELCAP and I-ELCAP studies provided information on the morphology and growth rate of early LC.

\section{National Lung Screening Trial (NLST)}

In 2011, the results of the NLST study were published, stating that LDCT screening led to $20 \%$ reduction of LC mortality as compared to CXR.

The baseline study found LC in $1 \%$ of participants and $62 \%$ of cancers were in stage I. This was the first randomized study that showed a significant reduction in LC mortality due to screening.

The NLST was conducted in 33 US centers from 2002 to $2007 ; 53,454$ participants were randomly assigned to LDCT or CXR. Participants received baseline screening and two annual rounds and were then subjected to observation (2).

The LDCT had four times higher diagnostic accuracy than CXR in detecting all LCs and about six times higher for stage I LC. At the same time, more false-positive diagnoses requiring invasive diagnostic procedures were made based on LDCT (7\% vs. 4\% of all participants) (37).

All NCNs $\geq 4 \mathrm{~mm}$, lung consolidation, obstructive atelectasis, growing nodules, and nodules with changes in attenuation were considered a positive result (38) directed for further investigation.

The threshold for nodules size in LDCT was $4 \mathrm{~mm}$ (Table 1). Positive predictive value (PPV) for the nodules $\geq 4 \mathrm{~mm}$ was $3.8 \%$, the same as overall PPV for this screening. For the smallest positive nodules (size of 4-6 mm) PPV was much lower-0.5\% and increased to $11.9 \%$ when nodules size went up to $11-20 \mathrm{~mm}$. PPV for each positive result as evaluated by a subsequent biopsy of the lesion was $52.9 \%$ (265 out of 501). Positive screen rates for the baseline and first annual round were similar $(27.3 \%$ vs. $27.9 \%$ ) and for the second round was lower (16.8\%) (39). The positive screen results in this trial depended only on nodule size (cut-off of $4 \mathrm{~mm}$ ) but not on nodule consistency.

In NLST, $40 \%$ of subjects had at least one falsepositive nodule that was not cancer over the three years of screening; 320 people had to undergo screening to prevent one death from LC; $96.4 \%$ of all positive results in the LDCT group were false positive (2) but $64 \%$ of them were due to small nodules (size of 4-7 $\mathrm{mm}$ ). In the retrospective analysis of the NLST population, when the nodule size threshold raises from 4 to $5 \mathrm{~mm}$ and $8 \mathrm{~mm}$, the proportion of missed or delayed cancers increased to $1 \%$ and $10.5 \%$, respectively and false-positive results were reduced by $15.8 \%$ and $65.8 \%$, respectively (40).

Despite such a significant number of false-positive results in NLST, data on a reduction in mortality prompted the U.S. Preventive Services Task Force (USPSTF) in December 2013 to issue a recommendation for LDCT as an effective screening tool for high-risk individuals. 


\section{Lung Imaging Reporting and Data System (Lung- RADS)}

With growing interest in LCS, the American College of Radiology (ACR) recognized the need to use a structured and simple decision-oriented system to perform and assess LDCT scans, and to manage and report detected pulmonary nodules. A diagnostic system based on nodule size, morphology, and growth rate was proposed by the ACR and named Lung-RADS. Lung-RADS was released in April 2014 in version 1.0 and upgraded to version 1.1. in April 2019 (41) and is currently widely used in the United States.

This system defines a positive screening test result and was created to reduce the percentage of false-positive results and limit unnecessary over-testing, radiation exposure, and harmful invasive procedures, as well as patient anxiety. Lung-RADS was created according to the model of other imaging assessment systems, of which BI-RADS has extensively been used in screening for breast cancer.

Both the Lung-RADS 1.0 and 1.1 system divide all nodules into 4 categories. Category 1 includes benign nodules with benign calcifications and fatty elements. Category 2 applies to benign appearance or behavior and includes among others solid nodules or nodules with a solid fraction with an average dimension $<6 \mathrm{~mm}$ or new nodules (which appeared in the next scan) with an average size $<4 \mathrm{~mm}$.

Category 3 is probably benign and has a low risk of malignancy (1-2\%). It includes solid or part-solid nodules with an average size $\geq 6 \mathrm{~mm}$ and less than $8 \mathrm{~mm}$ or with solid fraction $<6 \mathrm{~mm}$, and new solid nodules $\geq 4$ and $<6 \mathrm{~mm}$, than require a six-monthly LDCT check-up.

Category 4 concerns potentially malignant nodules and contains subgroups: $4 \mathrm{~A}, 4 \mathrm{~B}$, and $4 \mathrm{X}$ with a risk of malignancy from $5-15 \%$ for $4 \mathrm{~A}$ category to more than $15 \%$ for $4 \mathrm{~B}$ and $4 \mathrm{X}$ categories. In category 4 nodules an LDCT, biopsy, or PET-CT follow-up scan should be performed after 3 months (42).

The changes in version 1.1 of the Lung-RADS system are intended to reduce unnecessary diagnostics in benign lesions with minimal risk of LC development.

The perifissural nodules less than $10 \mathrm{~mm}$ were included in the category 2 of Lung-RADS 1.1 not requiring additional assessment, as the probability of malignancy in these lesions is $0(4,43,44)$. For intrapulmonary nodules $\geq 10 \mathrm{~mm}$ additional management based on the size criteria and lack of growth are necessary.
A new size criterion for non-solid nodules (GGNs) was also applied, increasing the size limit for category 2 from 20 to $30 \mathrm{~mm}$. Category 3 includes GGN $\geq 30 \mathrm{~mm}$ found on the baseline CT or newly detected GGN of any size in the annual round or unchanged or slowly-growing as compared to the previous examination; for more extensive growth or size, a nodule may be up-coded to $4 X$.

Category $4 \mathrm{~B}$ includes very suspicious new or growing solid $(\geq 8 \mathrm{~mm})$ and part-solid with solid component $(\geq 4 \mathrm{~mm})$ nodules which are detected in the annual screening which requiring a one-month LDCT follow-up to confirm infection.

Category $4 \mathrm{X}$ contains nodules which are moved up from other categories, based on additional potentially malignant findings (e.g., enlargement of lymph nodes). The probability of malignancy of $4 \mathrm{X}$ category was $46-57 \%$ and higher than other categories (45).

A reduction of LCS false-positive rates in Lung-RADS 1.1 in comparison to Lung-RADS 1.0 is possible by downgrading some categories, e.g., non-solid nodules of a diameter from 20 to $30 \mathrm{~mm}$ or that characterized by long VDT (mean VDT for growing NSNs is 769-1041) $(32,46)$.

The smaller nodules (category 2 ) that can be considered negative screen are followed by annual screening round in 12 months. The higher risk nodules (Categories 4B and 4X) go to more multidisciplinary management and additional testing.

According to the criteria of the Lung-RADS system, a positive result of LDCT imaging is considered to be a solid or part-solid nodule $\geq 6 \mathrm{~mm}$ or non-solid nodule $\geq 30 \mathrm{~mm}$ or a new solid nodule $\geq 4 \mathrm{~mm}$ (Table 2). The threshold size of $6 \mathrm{~mm}$ for the positive screen was implemented by LungRADS 1.0 in 2014 after the inclusion of these criteria in I-ELCAP. With these cut-off values for pulmonary nodules in a retrospective assessment, the percentage of falsepositive results in the NLST study would be much smaller; reaching $10.6 \%$ according to McKee and $12.8 \%$ according to Pinsky and sensitivity for baseline and annual rounds would reach $86.1 \%$ and $78.6 \%$ respectively $(47,48)$. This indicates that Lung-RADS criteria are more accurate for LC screening that NLST criteria.

\section{International Lung Screening trial (ILST)}

The ILST between 2017 and 2019 recruited asymptomatic volunteers at high risk of LC in Australia, Canada, and Hong Kong. Two LDCT screening rounds were planned: at baseline and after two years, with follow-up for LC 
Table 2 Different thresholds for negative, intermediate and positive screen at baseline according to guidelines

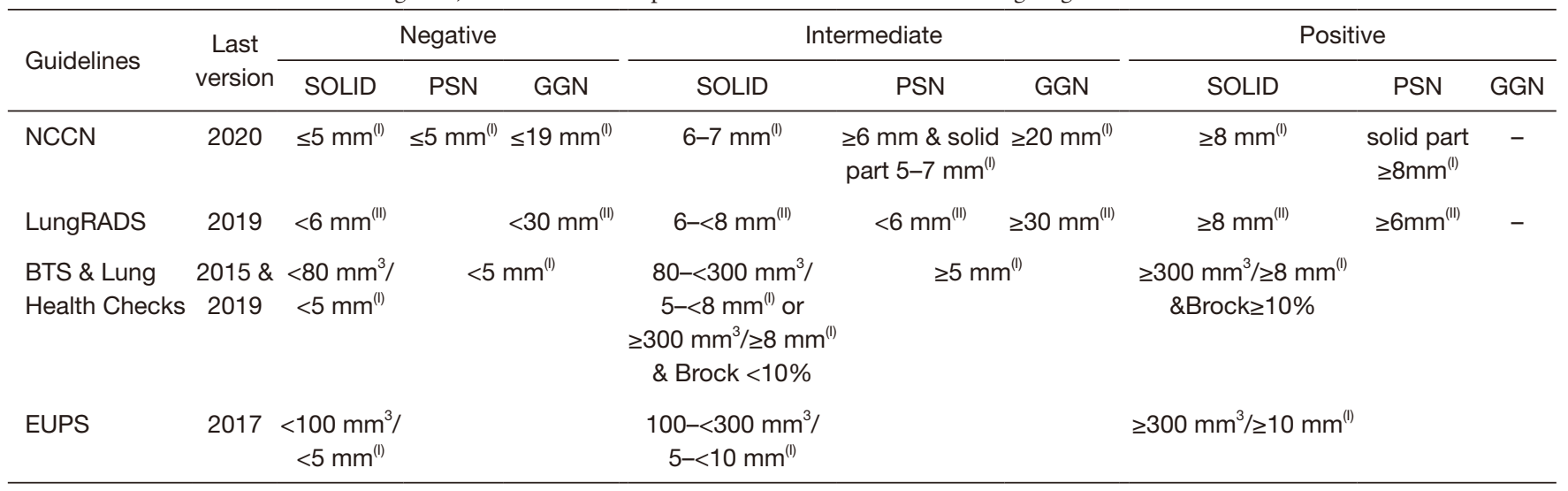

(I), maximum diameter; ${ }^{(I)}$, mean diameter.

outcomes over the next five years.

ILST aims to compare the efficiency of protocol based on PanCan nodule probability calculator with LungRADS and the United States Preventive Services Task Force (USPSTF) inclusion criteria with those based on the $\mathrm{PLCO}_{\mathrm{m} 2012}$ (Prostate, Lung, Colorectal, and Ovarian) Cancer Screening Trial model.

$\mathrm{PLCO}_{\mathrm{m} 2012}$ is a LC risk prediction model developed by Dr. Martin C. Tammegagi and colleagues to improve the identification of LC high-risk individuals who could benefit from LCS with proposed optimal risk threshold $1.51 \%$ over 6 years $(49,50)$.

The $\mathrm{PLCO}_{\mathrm{m} 2012}$ has higher sensitivity than the NLST criteria ( $83 \%$ vs. $71.4 \%$ ) without loss of specificity. The model uses well-established risk factors, such as age, smoking exposure, gender, race, ethnicity, education, body mass index (BMI), chronic obstructive pulmonary disease, emphysema, and a history of pneumonia or any other cancers. The PanCan (or Brock University) model is an alternative nodule management system based on individual cancer risk. For individual cancer risk estimation, regression modeling was used, where both information related to the tumor and the person are incorporated.

Nodules with LC risk calculated according to the PanCan model at baseline less than $1.5 \%$ are considered as normal findings (CAT1), $1.5 \%-<6 \%$ as low malignancy risk (CAT2), $6 \%-<30 \%$ as moderate malignancy risk (CAT3), $\geq 30 \%$ as high malignancy risk (CAT4). Individuals with non-infectious mass, mediastinal or hilar lymphadenopathy, or any size endobronchial nodule are assigned to CAT5 and qualified for biopsy similarly to CAT4 nodules (Table 1). CAT2 nodules are qualified for LDCT in one year, CAT1 in two years. CAT3 is divided into two subcategories CAT3a (PanCan score 6\% to 10\%) and CAT3b (PanCan score $10 \%$ to $30 \%$ ). For CAT3a an interval LDCT in 3 months is recommended, but for CAT3b, the treating physician decides on an interval LDCT or immediate clinical investigation. Significant nodule growth is more likely to be LC and needs further clinical evaluation. Significant interval growth depends on nodule size. For $5 \mathrm{~mm}$ nodules, it is an increase of $1.5 \mathrm{~mm}$ in average diameter or a volume change of $>100 \%$. For $5-10 \mathrm{~mm}$ nodules, it is defined as a volume change of $>30 \%$ and for nodules $>10 \mathrm{~mm}$, as a volume change of $>20 \%$. VDT as a predictor of malignancy is $30-400$ days for nodules $>300 \mathrm{~mm}^{3}$. The development of a solid component of $>6 \mathrm{~mm}$ in a subsolid nodule is a feature of significant interval growth.

Using LC risk prediction models for both identifying the screening group and for assessing detected noncalcified pulmonary nodules in the LDCT scan is an innovative approach for early detection of LC in high-risk individuals (51).

\section{NELSON}

The Dutch-Belgian Randomized Lung Cancer Screening Trial (Dutch acronym: NELSON study), the largest European randomized LCS study, has started in September 2003 with 3 years recruitment period. A total of 15,792 participants (7,900 in the CT arm and 7,892 in the control arm) between the age of 50 and 74 years old were randomly assigned to the CT and control arm $(52,53)$. The results of 10 years follow up were recently published and revealed a cumulative rate ratio (RR) for death from LC in men of 0,76 
$(\mathrm{P}=0.01)$ in the screening arm as compared with the control arm and $0.67 \mathrm{RR}$ among women (3).

NELSON study was the first large trial where semiautomated volume measurement of lung nodules with dedicated software was applied. The lung nodule assessment protocol included four screening rounds with CT scans in 1, 2, 4, and 6 years. Nodules detected at baseline screening were assigned to one of four nodule categories (NODCAT) based on their size: NODCAT I, II, III, or IV. For solid nodules, the 3D semi-automatic measurement was recorded by the radiologists. For partial solid nodules, the volume of the solid component and overall size of the nodule measured as the mean diameter of length and width $\left(\mathrm{d}_{\text {mean }}\right)$ were reported. Where the semiautomatic segmentation of the nodule failed, for example for non-solid lesions, 2-D manual measurement was applied $\left(\mathrm{d}_{\text {mean }}\right)$ while for the solid pleural lesions, the diameter perpendicular to the costal pleura was reported $\left(\mathrm{d}_{\min }\right)$.

Management of the nodules was a result of NODCAT category assignment at baseline round or nodule growth expressed by growth category type (GROWCAT) assessed according to VDT estimated in the following rounds of the screening.

Benign nodules containing fat or benign calcifications or other benign characteristics were counted as NODCAT I. NODCAT II category, called nonsignificantly small, contained nodules smaller than NODCAT III but of a different characteristic than NODCAT I. Individuals with NODCAT I and II-negative result-were planned for the next CT scan in one year. All solid nodules, size of $50-500 \mathrm{~mm}^{3}$, pleural based nodules size of $5-10 \mathrm{~mm}\left(\mathrm{~d}_{\min }\right)$ in short axis, non-solid nodules $\geq 8 \mathrm{~mm}\left(\mathrm{~d}_{\text {mean }}\right)$ and also partial solid nodules with the non-solid component: $\geq 8 \mathrm{~mm} \mathrm{~d}_{\text {mean }}$ or solid component: $50-500 \mathrm{~mm}^{3}$ were allocated to NODCAT III category-indeterminate test result which required a repeat scan in 3-4 month according to the protocol. NODCAT IV category-potentially malignant-included solid nodules $>500 \mathrm{~mm}^{3}$, solid-pleural based $>10 \mathrm{~mm}\left(\mathrm{~d}_{\min }\right)$ or partially solid with solid component $>500 \mathrm{~mm}^{3}$ (Table 1). Growing nodules with VDT $>600$ days were classified in GROWCAT A category (negative test), respectively nodules with VDT between 400-600 days-GROWCAT B (indeterminate test) and VDT $<400$ days-GROWCAT $\mathrm{C}$ (positive test). For new nodules detected in follow-up, the same cut-offs were used but the next CT exam was at a shorter interval (54).

The NELSON group reported that implementation of the volumetry and VDT based on the semiautomated assessment of detected nodules is more accurate than $2 \mathrm{D}$ measurement (55) and leads to a reduction in the number of false-positive tests, lower number of follow-up LDCTs and lower number of unnecessary diagnostic examinations (56) while preserving extremely low rate of interval cancers (52). The proportion of false positive CT scans in the NELSON trial was $1.2 \%$ as compared to $23.3 \%$ in the NLST study $(2,3)$.

\section{United Kingdom Lung Screen (UKLS)}

UKLS was a randomized controlled pilot trial that recruited high-risk individuals from 2011 to 2014. The first plan was to randomize 4,000 subjects to either LDCT or no screening with continuation to full study with randomization of further 28,000 subjects (57). Finally, UKLS has been completed with 4,055 participants (58). UKLS study plan was based on the Wald Single Screen Design. According to the protocol, one screening round was planned with 10 years follow up. This approach was the most economically reasonable and did not have a problem with long-term compliance. Men and women 50 to 75 years old with 5 -year LC risk of $\geq 5 \%$ according to the Liverpool Lung Project $\left(\mathrm{LLP}_{\mathrm{v} 2}\right)$ risk prediction model were randomly assigned to two study groups. UKLS was the first randomized LCS study where inclusion criteria were based on risk prediction model with LC risk calculated based not only on smoking history but also other factors like: age, sex, family history of LC, history of non-pulmonary malignant tumor, history of pneumonia and occupational exposure to asbestos (57) resulting in a higher percentage of LCs diagnosed at baseline comparing with NLST and NELSON trials (59). Such a risk model allows selecting the individuals at the highest risk of LC, therefore increasing screening effectiveness. In UKLS after baseline LDCT exam, detected nodules were assigned to one of four categories based on volume and their characteristic. Category 1 includednodules smaller than $15 \mathrm{~mm}^{3}$ or nodules containing fat or benign calcification. Intraparenchymal solid nodules with a volume of $15-49 \mathrm{~mm}^{3}$ were classified to category 2 with CT follow up in one year, nodules with a volume of $50-500 \mathrm{~mm}^{3}$ to category 3 with CT follow up in 3 and 9 month according to VDT, nodules with a volume $>500 \mathrm{~mm}^{3}$ to category 4 and were send directly for multidisciplinary team (MDT) assessment (Table 1). For solid pleural or juxta pleural and non-solid nodules, where the volume assessment was not possible, the linear measurement with certain cut offs for each category was taken. For part solid nodules the volume 
of solid component was calculated. During incidence CT further management was decided based on VDT. Nodules with VDT $<400$ days from category 2 in 1 year follow up CT and from category 3 in 3 or 9 month follow up CT were directed to MDT assessment. For individuals with multiple nodules, separate assessment of each nodule up to 20 per subject was performed (57).

During the UKLS pilot trial, $1.7 \%$ of participants at baseline and $2.1 \%$ of participants in total were diagnosed with LC. Among detected LCs $85.7 \%$ were stage I or II and $83.3 \%$ had surgical resection as their primary treatment with expected good 5 -year clinical outcome (60). The false positive rate reported in UKLS study was 3.6\% (58). The protocol developed in this study was also adapted for clinical use to manage incidentally detected nodules (57).

Based on UKLS data, the LC pulmonary nodule risk model was developed. This model includes such covariates like female gender, history of asthma, bronchitis, asbestos exposure, history of LC, early and late-onset of family history of LC, smoking duration, FVC (forced vital capacity), nodule type (pure ground-glass and part-solid) and what is novel, volume as measured by semiautomated volumetry. The UKLS Nodule Risk Model was created for future use in national CT screening programs with protocols based on the volumetric assessment of detected lung nodules (61).

\section{British Thoracic Society (BTS)}

The BTS Guideline Development Group (BTS GDG) has decided to summarize the previous experience and reports from literature as well as expert opinions and has proposed updated, comprehensive recommendations for the management of pulmonary nodules in 2015 . These guidelines are endorsed by the European Society of Radiology (ESR) and European Society of Thoracic Imaging (ESTI) as well as LungRads and EUPS (European Union position statement). BTS guidelines included the same recommendations for incidentally and screeningdetected nodules. In these very detailed guidelines, volumetry as a preferred measurement method was proposed. When the measurement of the volumetry is not possible, the longest diameter measured on axial scans is acceptable. For individuals with multiple nodules, the management algorithm is determined according to the largest one.

BTS GDG does not recommend follow-up of solid nodules with benign features, i.e. containing fat or benign calcifications, nodules smaller than $80 \mathrm{~mm}^{3}$ or $<5 \mathrm{~mm}$ in maximum diameter, and also typical perifissural or subpleural nodules smaller than $10 \mathrm{~mm}$. For solid nodules $\geq 80 \mathrm{~mm}^{3}$ and smaller than $<300 \mathrm{~mm}^{3}$, surveillance CT in 3 months after the baseline is proposed. The same management is proposed for nodules $\geq 6$ and $<8 \mathrm{~mm}$ if assessed by linear measurement. The next steps are based on VDT: if $\leq 400$ days-growth typical for malignant nodules-further workup and consideration of definitive management is needed. For nodules with VDT $>400$ after surveillance CT and for nodules $5-6 \mathrm{~mm}$ in diameter in baseline CT, next CT in 1 year after the baseline is recommended. The next steps are taken based on the result of VDT. For nodules with VDT $\leq 400$ days, definitive management should be considered. For nodules with VDT =400-600 days, biopsy should be considered or further CT surveillance depending on patient preference. Stable or slowly growing nodules with VDT $>600$ days can be discharged or if slowly growing based on volumetry the surveillance CT depending on patient preference can be offered. If the nodule is stable based on 2D non-automated diameter, CT in two years from baseline should be performed. For nodules $\geq 300 \mathrm{~mm}^{3}$ or $\geq 8 \mathrm{~mm}$ in diameter at baseline CT, assessment of LC risk using Brock model is recommended. When the risk of malignancy is $\geq 10 \%$, further evaluation with PET-CT is recommended and Herder risk model calculation necessary for deciding about treatment, biopsy, or further surveillance.

BTS GDG elaborated a separate algorithm for the management of sub-solid nodules (SSNs) defined as partsolid (PSN) or pure pGGN. They do not recommend follow-up SSNs $<5 \mathrm{~mm}$ in maximum diameter at baseline. For SSNs $\geq 5 \mathrm{~mm}$ repeat CT in 3 months is recommended to assess growth. For growing nodules or nodules with a new solid component, resection or non-surgical treatment is recommended. For stable nodules risk of malignancy should be assessed according to morphology and based on Brock model. For nodules with a risk of malignancy $>10 \%$ according to Brock model and depending on patient preference, different approaches can be selected: surveillance CT in 1, 2, 4 years from baseline, image-guided biopsy, resection, or non-surgical treatment (62).

In compliance with BTS guidelines, in January 2019 Targeted Screening for Lung Cancer with Low Radiation Dose Computed Tomography Standard Protocol was prepared for the Targeted Lung Health Checks Programme (63). In this algorithm, the same size thresholds for nodules detected at baseline CT like in BTS guidelines 
were implemented (i.e., 80 and $300 \mathrm{~mm}^{3}$ ) (Table 2). For individuals with no nodules or with nodules smaller than $80 \mathrm{~mm}^{3}$ (<5 mm max. diameter) at baseline scan, the next CT in 24 months was recommended. For nodules $\geq 80$ and $<300 \mathrm{~mm}^{3}$, CT in 3 and 12 months was recommended. In case when the assessment of volumetry is not possible and the maximum diameter on axial scans is evaluated, different thresholds with more frequent and longer follow up are applied. For nodules measuring 5 to $6 \mathrm{~mm}$ in max. diameter CT scan in 12 and 24 months should be performed. For bigger nodules measuring $\geq 6$ and $<8 \mathrm{~mm}$ follow up at 3 , 12 and 24 months should be done. Nodules $\geq 300 \mathrm{~mm}^{3}$ or $\geq 8 \mathrm{~mm}$ in max. the diameter should be evaluated with Brock risk model. Nodules with LC risk $\geq 10 \%$ should be referred for MDT assessment. Nodules with LC risk $<10 \%$ qualify for CT in 3 and 12 months when assessed with volumetry, and 3,12 and 24 months when the size is expressed by linear measurement.

Concerning a possible malignant etiology of the nodules found on any interval CT, different cut-offs of $30 \mathrm{~mm}^{3}$ (4 mm max. diam.) and $300 \mathrm{~mm}^{3}$ (8 mm max. diameter) and also, slightly different intervals for follow up were proposed. Nodules $\geq 30$ and $<300 \mathrm{~mm}^{3}$ should be assessed in CT at 3 and 12 months, $\geq 4$ and $<8 \mathrm{~mm}$ in 3,12 , and 24 months. Nodules $\geq 300 \mathrm{~mm}^{3}(\geq 8 \mathrm{~mm})$ should be referred for MDT assessment. No change in follow up is recommended for nodules $<30 \mathrm{~mm}^{3}$ ( $<4 \mathrm{~mm}$ max. diam.) A targeted LCS program was designed to reduce mortality from LC. This described in detail project aims to select participants from a local population at high risk of LC and offer LDCT to the eligible individuals (63).

The demonstration program scheduled to start in 2020 in Poland will implement modified criteria for the management of solid pulmonary nodules based on the protocol described above.

\section{EUPS on LCS}

The EUPS published in 2017, summarized major issues concerning the successful implementation of low-dose CT LCS in Europe. In these recommendations, a separate approach for nodules detected at baseline screening and newly detected lung nodules in the next screening rounds were made. For nodules detected outside of screening programs, BTS guidelines were implemented. In LCS setting for solid nodules, volumetric assessment based on semi-automated measurement was recommended as a more precise and reproducible method. The manual measurement of maximum diameter was accepted if volumetry would not be technically possible. At baseline screening, cut-off volume values of 100 and $300 \mathrm{~mm}^{3}$ were proposed (59) (Table 2) based on LC probability results of the first two screening rounds from the NELSON trial (64). Individuals with solid non-calcified nodules with clear features of benign disease and solid nodules $<100 \mathrm{~mm}^{3}$ or $<5 \mathrm{~mm}$ in diameter should be sent to the next round of the screening according to the protocol. For solid nodules with a volume of 100 to $300 \mathrm{~mm}^{3}$ or 5 to $<10 \mathrm{~mm}$ in diameter, a repeat screen in 3 months should be performed. Further management should be based on VDT. If VDT $>600$ days the next assessment should be performed in the next round of the screening according to the protocol. Further workup and consideration of definitive management according to a MDT decision should be foreseen for nodules with VDT $\leq 600$ days after CT control scan at 3 months and solid nodules size of $\geq 300 \mathrm{~mm}^{3}$ or $\geq 10 \mathrm{~mm}$ at baseline CT. The 600 days cut-off for VDT was proposed according to the data provided by the NELSON group (64). A separate approach was proposed for the new incident nodules detected after baseline screening as potentially malignant, fast-growing with a high risk of LC of $2.8 \%$ according to the literature $(8,14,64,65)$. These nodules should be categorized according to the volume as $<30 \mathrm{~mm}^{3}$ ( $<4 \mathrm{~mm}$ ), $30-200 \mathrm{~mm}^{3}$ ( 4 to $<8 \mathrm{~mm}$ ), and $\geq 200 \mathrm{~mm}^{3}$ ( $\geq 8 \mathrm{~mm}$ ). For the first category, the next round of the screening should be scheduled. The second category should be evaluated with LDCT again in 3 months. If VDT exceeds 600 days -return to the next round of the screening should take place, if VDT $\leq 600$ days further workup should be performed after the decision of MDT. Nodules from the third category $\left(\geq 200 \mathrm{~mm}^{3}\right)$ should be scheduled for further workup as potentially malignant. Management of SSNs should be performed according to the BTS guidelines and surveillance should be considered as a preferred procedure to avoid overdiagnosis. The EU position statement did not recommend any specific risk prediction model among those listed in the statement but underlined that future LCS programs should use a validated risk stratification approach to select for screening program individuals at high risk of LC. Also, the final decision about the optimal timing of LCS intervals was postponed according to mixed data from previous screening trials with only evidence for annual lowdose CT and recommendation that a more personalized approach based on baseline and first screening rounds may be considered (59).

\section{Conclusions}


Nodule management protocols in LCS have substantially evolved over the last two decades due to technological advances and better selection of cut-off points to define the risk of malignancy. These advances reduced the risk of unnecessary diagnostic and invasive procedures while preserving the utility of LDCT to diagnose early LC and decrease LC mortality. Based on the experience from multiple screening programs the radiological decision should include individual risk assessment, automated or semiautomated nodule volume evaluations, calculation of volume doubling time. Optimal selection of screening interval is also crucial but for now, there is no consensus whether it should be annual screening interval or biennial in some individuals with negative baseline result. The management algorithms for new nodules detected at subsequent rounds of screening were proposed and are now routinely implemented in the new generation of LCS interventions, with hopes for its widespread application.

\section{Acknowledgments}

Funding: None.

\section{Footnote}

Provenance and Peer Review: This article was commissioned by the Guest Editor (Witold Rzyman) for the series "Implementation of CT-based screening of lung cancer" published in Translational Lung Cancer Research. The article has undergone external peer review.

Peer Review File: Available at http://dx.doi.org/10.21037/ tlcr-20-755

Conflicts of Interest: All authors have completed the ICMJE uniform disclosure form (available at http://dx.doi. org/10.21037/tlcr-20-755). The series "Implementation of CT-based screening of lung cancer" was commissioned by the editorial office without any funding or sponsorship. The authors have no other conflicts of interest to declare.

Ethical Statement: The authors are accountable for all aspects of the work in ensuring that questions related to the accuracy or integrity of any part of the work are appropriately investigated and resolved.

Open Access Statement: This is an Open Access article distributed in accordance with the Creative Commons
Attribution-NonCommercial-NoDerivs 4.0 International License (CC BY-NC-ND 4.0), which permits the noncommercial replication and distribution of the article with the strict proviso that no changes or edits are made and the original work is properly cited (including links to both the formal publication through the relevant DOI and the license). See: https://creativecommons.org/licenses/by-nc-nd/4.0/.

\section{References}

1. American Cancer Society. Cancer Facts \& Figures 2018.

2. Aberle DR, Adams AM, Berg CD, et al. Reduced lungcancer mortality with low-dose computed tomographic screening. N Engl J Med 2011;365:395-409.

3. de Koning HJ, Van Der Aalst CM, De Jong PA, et al. Reduced lung-cancer mortality with volume CT screening in a randomized trial. N Engl J Med 2020;382:503-13.

4. Hansell DM, Bankier AA, MacMahon H, et al. Fleischner Society: Glossary of terms for thoracic imaging. Radiology 2008;246:697-722.

5. Rzyman W, Szurowska E, Adamek M. Implementation of lung cancer screening at the national level: Polish example. Transl Lung Cancer Res 2019;8:S95-105.

6. MacMahon H, Naidich DP, Goo JM, et al. Guidelines for management of incidental pulmonary nodules detected on CT images: From the Fleischner Society 2017. Radiology 2017;284:228-43.

7. Henschke CI, Miettinen OS, Yankelevitz DF, et al. Radiographic screening for cancer proposed paradigm for requisite research. Clin Imaging 1994;18:16-20.

8. Henschke CI, Naidich DP, Yankelevitz DF, et al. Early Lung Cancer Action Project: Initial Findings on Repeat Screenings. Cancer 2001;92:153-9.

9. Henschke CI, Yankelevitz DF, Naidich DP, et al. CT Screening for Lung Cancer: Suspiciousness of Nodules according to Size on Baseline Scans. Radiology 2004;231:164-8.

10. Henschke CI, McCauley DI, Yankelevitz DF, et al. Early Lung Cancer Action Project: Overall design and findings from baseline screening. Lancet 1999;354:99-105.

11. Li K, Yip R, Avila R, et al. Size and Growth Assessment of Pulmonary Nodules: Consequences of the Rounding. J Thorac Oncol 2017;12:657-62.

12. Henschke CI, Yankelevitz DF, McCauley DI, Rifkin M, Fiore ES, Austin JHM, et al. CT screening for lung cancer: Diagnoses resulting from the New York Early Lung Cancer Action Project. Radiology 2007;243:239-49.

13. Evolution of Lung Cancer Screening Management - 
PubMed [Internet]. [cited 2020 May 16]. Available online: https://pubmed.ncbi.nlm.nih.gov/31365749/

14. Henschke CI, Yankelevitz DF, Libby DM, et al. Survival of patients with stage I lung cancer detected on CT screening. N Engl J Med 2006;355:1763-71.

15. Henschke CI, Yankelevitz DF, Mirtcheva R, et al. CT screening for lung cancer: Frequency and significance of part-solid and nonsolid nodules. AJR Am J Roentgenol 2002;178:1053-7.

16. Henschke CI, Shaham D, Yankelevitz DF, et al. CT screening for lung cancer: Significance of diagnoses in its baseline cycle. Clin Imaging 2006;30:11-5.

17. Henschke CI, Yip R, Yankelevitz DF, et al. Definition of a positive test result in computed tomography screening for lung cancer. Ann Intern Med 2013;158:246-52.

18. Yip R, Henschke CI, Yankelevitz DF, et al. Screening for lung cancer: Alternative definitions of positive test result based on the national lung screening trial and international early lung cancer action program databases. Radiology 2014;273:591-6.

19. Yankelevitz DF, Yip R, Smith JP, et al. CT screening for lung cancer: Nonsolid nodules in baseline and annual repeat rounds. Radiology 2015;277:555-64.

20. Available online: www.ielcap.org

21. Duffy SW, Tabar L, Fagerberg G, et al. Breast screening, prognostic factors and survival - Results from the Swedish two county study. Br J Cancer 1991;64:1133-8.

22. Carter D, Vazquez M, Flieder DB, et al. Comparison of pathologic findings of baseline and annual repeat cancers diagnosed on CT screening. Lung Cancer 2007;56:193-9.

23. Henschke CI, Yankelevitz DF, Yip R, et al. Lung cancers diagnosed at annual CT screening: Volume doubling times. Radiology 2012;263:578-83.

24. Reeves AP, Chan AB, Yankelevitz DF, et al. On measuring the change in size of pulmonary nodules. IEEE Trans Med Imaging 2006;25:435-50.

25. Yankelevitz DF, Reeves AP, Kostis WJ, et al. Small pulmonary nodules: Volumetrically determined growth rates based on CT evaluation. Radiology 2000;217:251-6.

26. Yankelevitz DF, Gupta R, Zhao B, et al. Small pulmonary nodules: Evaluation with repeat CT - Preliminary experience. Radiology 1999;212:561-6.

27. Henschke CI, Yankelevitz DF, Yip R, et al. Tumor volume measurement error using computed tomography imaging in a phase II clinical trial in lung cancer. J Med Imaging (Bellingham) 2016;3:035505.

28. Kostis WJ, Yankelevitz DF, Reeves AP, et al. Small Pulmonary Nodules, Reproducibility of Three- dimensional Volumetric Measurement and Estimation of Time to Follow-up CT. Radiology 2004;231:446-52.

29. Henschke CI, Yankelevitz DF, Miettinen OS. Computed tomographic screening for lung cancer: The relationship of disease stage to tumor size. Arch Intern Med 2006;166:321-5.

30. Austin JHM, Yip R, D'Souza BM, et al. Small-cell carcinoma of the lung detected by CT screening: Stage distribution and curability. Lung Cancer 2012;76:339-43.

31. Libby DM, Wu N, Lee IJ, et al. CT screening for lung cancer: The value of short-term CT follow-up. Chest 2006;129:1039-42.

32. de Hoop B, Gietema H, Van De Vorst S, et al. Pulmonary ground-glass nodules: Increase in mass as an early indicator of growth. Radiology 2010;255:199-206.

33. Yankelevitz DF, Chan C, Henschke CI. Overdiagnosis: "A Malformed Concept". J Thorac Imaging 2019;34:151-3.

34. Flores R, Bauer T, Aye R, et al. Balancing curability and unnecessary surgery in the context of computed tomography screening for lung cancer. J Thorac Cardiovasc Surg 2014;147:1619-26.

35. Travis WD, Brambilla E, Burke AP, et al. Introduction to the 2015 World Health Organization Classification of Tumors of the Lung, Pleura, Thymus, and Heart. J Thorac Oncol 2015;10:1240-2.

36. Detterbeck FC, Boffa DJ, Kim AW, et al. The Eighth Edition Lung Cancer Stage Classification. Chest 2017;151:193-203.

37. Croswell JM, Baker SG, Marcus PM, et al. Cumulative incidence of false-positive test results in lung cancer screening: A randomized trial. Ann Intern Med 2010;152:505-12.

38. National Lung Screening Trial Research Team, Aberle DR, Berg CD, et al. The national lung screening trial: Overview and study design. Radiology 2011;258:243-53.

39. Church TR, Black WC, Aberle DR, et al. Results of initial low-dose computed tomographic screening for lung cancer. N Engl J Med 2013;368:1980-91.

40. Gierada DS, Pinsky P, Nath H, et al. Projected outcomes using different nodule sizes to define a positive CT lung cancer screening examination. J Natl Cancer Inst 2014;106:1-7.

41. Available online: www.acr.org

42. Available online: www.acr.org/-/media/ACR/Files/RADS/ Lung-RADS/LungRADSAssessmentCategoriesv1-1. pdf?la=en

43. de Hoop B, Van Ginneken B, Gietema H, et al. Pulmonary perifissural nodules on CT scans: Rapid growth is not a 
predictor of malignancy. Radiology 2012;265:611-6.

44. Ahn MI, Gleeson TG, Chan IH, et al. Perifissural nodules seen at CT screening for lung cancer. Radiology 2010;254:949-56.

45. Chung K, Jacobs C, Scholten ET, et al. Lung-RADS Category 4X: Does It Improve Prediction of Malignancy in Subsolid Nodules? Radiology 2017;284:264-71.

46. Veronesi G, Travaini LL, Maisonneuve P, et al. Positron emission tomography in the diagnostic workup of screening-detected lung nodules. Eur Respir J 2015;45:501-10.

47. McKee BJ, Regis SM, McKee AB, et al Performance of ACR lung-RADS in a clinical CT lung screening program. J Am Coll Radiol 2015;12:273-6.

48. Pinsky PF, Gierada DS, Black W, et al. Performance of lung-RADS in the national lung screening trial: A retrospective assessment. Ann Intern Med 2015;162:485-91.

49. Tammemägi MC, Katki HA, Hocking WG, et al. Selection criteria for lung-cancer screening. N Engl J Med 2013;368:728-36.

50. Tammemägi MC, Church TR, Hocking WG, et al. Evaluation of the Lung Cancer Risks at Which to Screen Ever- and Never-Smokers: Screening Rules Applied to the PLCO and NLST Cohorts. PLoS Med 2014;11:e1001764.

51. Lim KP, Marshall H, Tammemägi $M$, et al. CLINICAL STUDY DESIGN Protocol and Rationale for the International Lung Screening Trial Clinical Study Design 503 Background. Ann Am Thorac Soc 2020;17:503-12.

52. Ru Zhao Y, Xie X, de Koning HJ, et al. NELSON lung cancer screening study. Cancer Imaging 2011;11 Spec No A:S79-84.

53. Kauczor HU, Baird AM, Blum TG, et al. ESR/ERS statement paper on lung cancer screening. Eur Respir J 2020;55:1900506.

54. Xu DM, Gietema H, de Koning H, et al. Nodule management protocol of the NELSON randomised lung cancer screening trial. Lung Cancer 2006;54:177-84.

55. Heuvelmans MA, Vliegenthart R, de Koning HJ, et al. Quantification of growth patterns of screen-detected lung cancers: The NELSON study. Lung Cancer 2017;108:48-54.

Cite this article as: Dziadziuszko K, Szurowska E. Pulmonary nodule radiological diagnostic algorithm in lung cancer screening. Transl Lung Cancer Res 2021;10(2):1124-1135. doi: 10.21037/tlcr-20-755
56. van Klaveren RJ, Oudkerk M, Prokop M, et al. Management of lung nodules detected by volume CT scanning. N Engl J Med 2009;361:2221-9.

57. Baldwin DR, Duffy SW, Wald NJ, et al. UK Lung Screen (UKLS) nodule management protocol: Modelling of a single screen randomised controlled trial of low-dose CT screening for lung cancer. Thorax 2011;66:308-13.

58. Field JK, Duffy SW, Baldwin DR, et al. The UK lung cancer screening trial: A pilot randomised controlled trial of low-dose computed tomography screening for the early detection of lung cancer. Health Technol Assess 2016;20:1-146.

59. Oudkerk M, Devaraj A, Vliegenthart R, et al. European position statement on lung cancer screening. Lancet Oncol 2017;18:e754-66.

60. Field JK, Duffy SW, Baldwin DR, et al. UK Lung Cancer RCT Pilot Screening Trial: Baseline findings from the screening arm provide evidence for the potential implementation of lung cancer screening. Thorax 2016;71:161-70.

61. Marcus MW, Duffy SW, Devaraj A, et al. Probability of cancer in lung nodules using sequential volumetric screening up to 12 months: The UKLS trial. Thorax 2019;74:761-7.

62. Callister MEJ, Baldwin DR, Akram AR, et al. British Thoracic Society guidelines for the investigation and management of pulmonary nodules. Thorax 2015;70 Suppl 2:ii1-ii54.

63. NHS England, Reader I. Targeted Screening for Lung Cancer with Low Radiation Dose Computed Tomography. Standard Protocol prepared for the Targeted Lung Health Checks Programme. 2019.

64. Horeweg N, van Rosmalen J, Heuvelmans MA, et al. Lung cancer probability in patients with CT-detected pulmonary nodules: A prespecified analysis of data from the NELSON trial of low-dose CT screening. Lancet Oncol 2014;15:1332-41.

65. Swensen SJ, Jett JR, Sloan JA, et al. Screening for lung cancer with low-dose spiral computed tomography. Am J Respir Crit Care Med 2002;165:508-13. 